\title{
THE MINKOWSKI UNITS OF RIBBON KNOTS
}

\section{J. J. ANDREWS AND FORREST DRISTY 1}

1. Introduction. Following Fox [1], we define a ribbon to be a singular disk in $R^{3}$ in which all the singularities are of the type shown in Figure 1. A knot is a ribbon knot if it is the boundary of a ribbon.
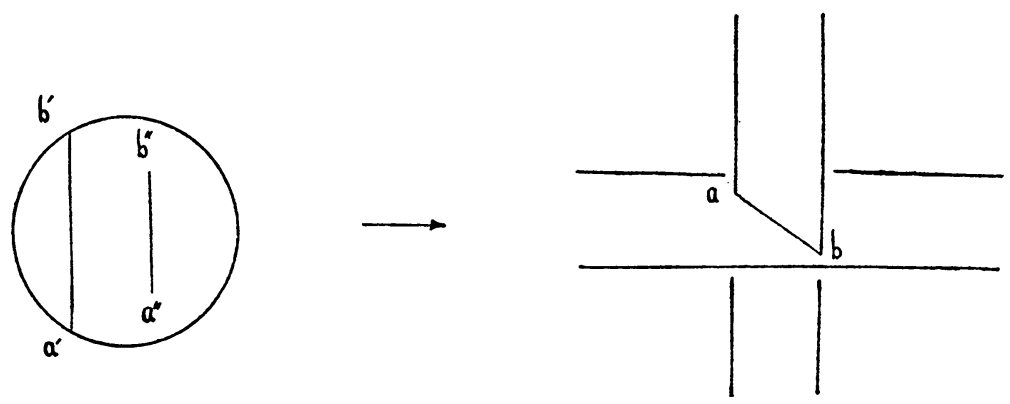

Figure 1

For each prime integer $p>2$, a unit $C_{p}= \pm 1$, called a Minkowski unit $[2$, p. 28], can be assigned to a given knot projection. These units are knot invariants. The main result of this paper is that for ribbon knots these units are all +1 .

2. Ribbon projections. Let $k$ be a ribbon knot in $R^{3}$, let $I^{2}$ be the unit square, and let $f: I^{2} \rightarrow R^{3}$ be a ribbon whose boundary is $k$. By definition of a ribbon, the singular set $S=\left\{x \mid f^{-1} f(x) \neq x\right\}$ of $f$ consists of an even number $2 n$ of pairwise disjoint arcs. Half of these arcs, say $C_{1}, \cdots, C_{n}$, have the property that both their endpoints lie on $\dot{I}^{2}$, but they are otherwise disjoint from $\dot{I}^{2}$. For each $C_{i}, i=1, \cdots, n$, there is a unique arc $C_{i}^{\prime}$ in $S$ such that $f\left(C_{i}^{\prime}\right)=f\left(C_{i}\right)$ and $C_{i}^{\prime} \cap \dot{I}^{2}=\varnothing$. The arcs $C_{i}$ separate $I^{2}$ into $n+1$ components $X_{0}, \cdots, X_{n}$, and the endpoints of the arcs $C_{i}$ separate $\dot{I}^{2}$ into a number of component arcs. At least two of these component arcs, say $E_{1}, \cdots, E_{m}$, have the property that they join the two endpoints of some arc $C_{i}$.

For each $i=1, \cdots, n$, let $p_{i}$ be an interior point of $C_{i}$ and let $p_{i}^{\prime}$ be the unique point of $C_{i}^{\prime}$ such that $f\left(p_{i}^{\prime}\right)=f\left(p_{i}\right)$. Also let $q_{i}$ be an interior point of $E_{i}$ for each $i=1, \cdots, m$. Then each component $X_{i}$ of $I^{2}-U_{i=1}^{n} C_{i}$ has a certain number $r$ of $p_{i}^{\prime}$ 's and $q_{i}^{\prime}$ 's designated on its boundary and a certain number $s$ of $p_{i}^{\prime}$ 's designated on its interior.

Presented to the Society January 24, 1963; received by the editors February 25, 1963, and, in revised form, July 10, 1963.

1 This research was supported by NSF Grant G-14089. 
It is easy to prove, by induction on the sum $r+s$, that in each $X_{i}$ there is a tree $T_{i}$ such that

(1) $T_{i} \cap \dot{X}_{i}$ consists of the $r$ points designated on $\dot{X}_{i}$.

(2) $T_{i}$ intersects the $\operatorname{arcs} C_{i}^{\prime}$ contained in $X_{i}$ only in the designated points $p_{i}^{\prime}$.

(3) All branch points of $T_{i}$ are of order 3 and are different from the points $p_{i}^{\prime}$.

(4) The points $p_{i}$ are the endpoints of the tree $T_{i}$.

(5) The points $p_{i}^{\prime}$ are piercing points of the $\operatorname{arcs} C_{i}^{\prime}$, i.e., osculating points are not admitted.

See Figure 2.

Since no two of the trees $T_{i}$ intersect in more than one point, the union $T=\mathrm{U}_{i=0}^{n} T_{i}$ is also a tree and has the properties:

(1) $T \cap \dot{I}^{2}=\left\{q_{1}, \cdots, q_{m}\right\}$,

(2) $T \cap C_{i}=p_{i}$,

(3) $T \cap C_{i}^{\prime}=p_{i}^{\prime}$.

See Figure 2.

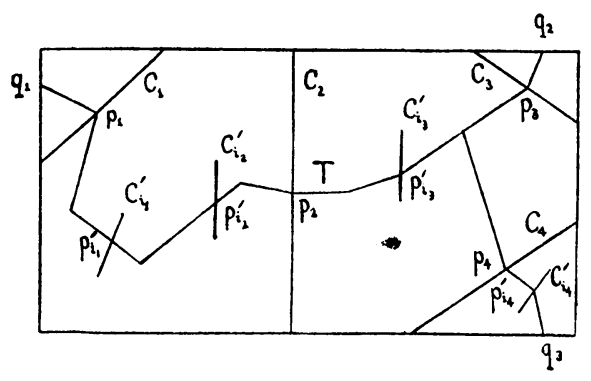

FIGURE 2

Now $I^{2} \searrow T \cup \cup_{i-1}^{n}\left(C_{i} \cup C_{i}^{\prime}\right)$, where the symbol $A \searrow B$ means " $A$ collapses to $B$ by Whitehead elementary collapsings" [3]. Hence

$$
f\left(I^{2}\right) \searrow f\left[T \cup \bigcup_{i=1}^{n}\left(C_{i} \cup C_{i}^{\prime}\right)\right]=f(T) \cup f\left(\bigcup_{i=1}^{n} C_{i}\right)
$$

and since

$$
f(T) \cup f\left(\bigcup_{i=1}^{n} C_{i}\right) \searrow f(T)
$$

it follows that

$$
f\left(I^{2}\right) \searrow f(T) .
$$

If $\epsilon$ is a positive real number, let $N(f(T), \epsilon)$ represent a regular 
neighborhood of $f(T)$ which lies within the $\epsilon$-neighborhood of $f(T)$.

LEMMA 1. For each $\epsilon>0$ and for each $N(f(T), \epsilon)$ there is a ribbon $g: I^{2} \rightarrow R^{3}$ such that

$$
F(T) \subset g\left(I^{2}\right) \subset f\left(I^{2}\right) \cap N(f(T), \epsilon)
$$

and such that $g\left(\dot{I}^{2}\right)$ and $f\left(\dot{I}^{2}\right)$ are knots of the same type.

Proof. Since $f$ is a continuous function defined on a compact set, it is uniformly continuous and hence for each $\epsilon>0$ and for each $N(f(T), \epsilon)$ there exists a $\delta>0$ such that if $x \in I^{2}$ and $d(x, T)<\delta$, then $f(x) \in N(f(T), \epsilon)$. For each $i=1, \cdots, n$ let $a_{i}^{\prime}$ and $b_{i}^{\prime}$ be distinct points of $C_{i}^{\prime}$ such that $p_{i}^{\prime} \in a_{i}^{\prime} b_{i}^{\prime} \subset N(T, \delta)$, and let $a_{i}$ and $b_{i}$ be points of $C_{i}$ such that $f\left(a_{i}\right)=f\left(a_{i}^{\prime}\right)$ and $f\left(b_{i}\right)=f\left(b_{i}^{\prime}\right)$. For each $i=1, \cdots, n$ let $a_{i}^{\prime \prime}$ and $b_{i}^{\prime \prime}$ be distinct points of $C_{i} \cap N(T, \delta)$ such that $p_{i} \in a_{i}^{\prime \prime} b_{i}^{\prime \prime}$ and such that $a_{i}^{\prime \prime} b_{i}^{\prime \prime} \subset$ Int $a_{i} b_{i}$. For $i=1, \cdots, m$ let $c_{i}$ and $d_{i}$ be points of $E_{i} \cap N(T, \delta)$ such that $q_{i} \in c_{i} d_{i}$. Then there is a regular neighborhood $D$ of $T$ such that

(i) $a_{i}^{\prime \prime} b_{i}^{\prime \prime} \subset D$ and $\dot{D} \cap C_{i}=\left\{a_{i}^{\prime \prime}, b_{i}^{\prime \prime}\right\}$ for $i=1, \cdots, n$,

(ii) $a_{i}^{\prime} b_{i}^{\prime} \subset D$ and $\dot{D} \cap C_{i}^{\prime}=\left\{a_{i}^{\prime}, b_{i}^{\prime}\right\}$ for $i=1, \cdots, n$,

(iii) $c_{i} d_{i} \subset D$ and $\dot{D} \cap E_{i}=\left\{c_{i}, d_{i}\right\}$ for $i=1, \cdots, m$.

See Figure 3.

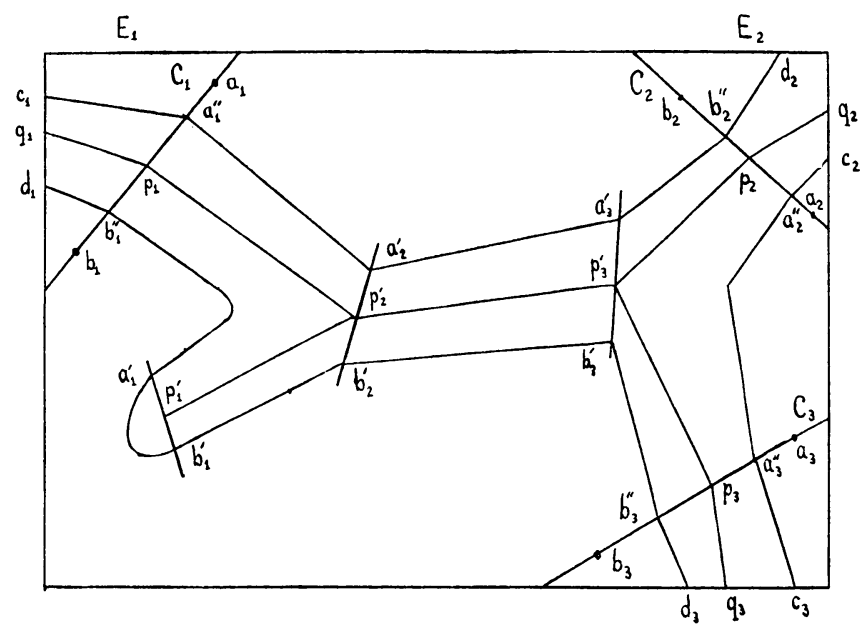

FIGURE 3

Let $h: I^{2} \rightarrow D$ be a homeomorphism and let

$$
g=f \circ h: I^{2} \rightarrow f\left(I^{2}\right) .
$$

Then $f(T) \subset g\left(I^{2}\right) \subset f\left(I^{2}\right) \cap N(f(T), \epsilon)$. 
To see that $f\left(\dot{I}^{2}\right)$ and $g\left(\dot{I}^{2}\right)$ are knots of the same type, we observe that together they bound a singular surface which collapses to either of them. Such a collapsing gives an isotopy of one of these knots onto the other, and this completes the proof of the lemma.

Let $P: R^{3} \rightarrow R^{2}$ be a projection such that $P$ is one-to-one on $f(T)$ except for a finite number of doublepoints, and such that no vertex of $f(T)$ is projected onto a doublepoint. If $\epsilon$ is chosen sufficiently small, the above lemma provides us with a ribbon $g\left(I^{2}\right)$ whose boundary $g\left(\dot{I}^{2}\right)$ is of the same knot type as the given knot $k=f\left(\dot{I}^{2}\right)$ and such that its projection $P\left(g\left(I^{2}\right)\right)$ has no singularities except those resulting from doublepoints in $P(f(T))$; that is, singularities of the forms
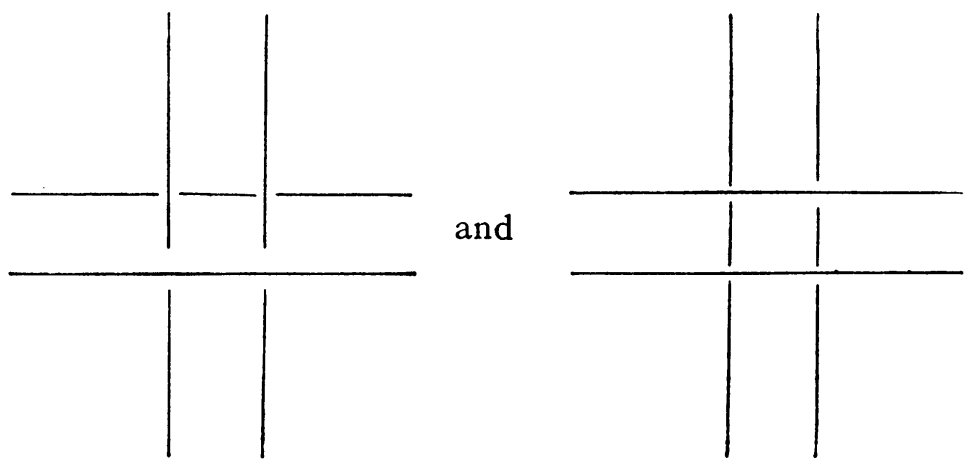

and singularities resulting from "twists" in $g\left(I^{2}\right)$; that is, singularities of the form

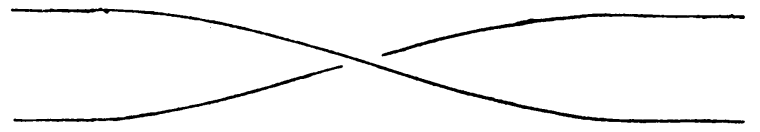

Restricting $P$ to $g\left(\dot{I}^{2}\right)$ gives a knot projection which we shall call a ribbon projection of the knot $k$. A ribbon projection will be called twist-free if it has no crossings of the form

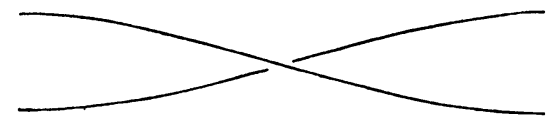

THEOREM 2. Every ribbon knot has a twist-free ribbon projection.

Proor. Let $P$ be an arbitrary ribbon projection of a ribbon knot $k$. We shall show that if $P$ is not twist-free, the twists in it can be moved out to the ends of the ribbon and thus be removed. The ribbon can pass over or through itself in only four essentially different ways, and we need only show that a twist can be moved past each type of 
crossing and also past a "fork" in the ribbon. The following diagrams show how each of these cases can be handled, moving the twist to the right in each case.
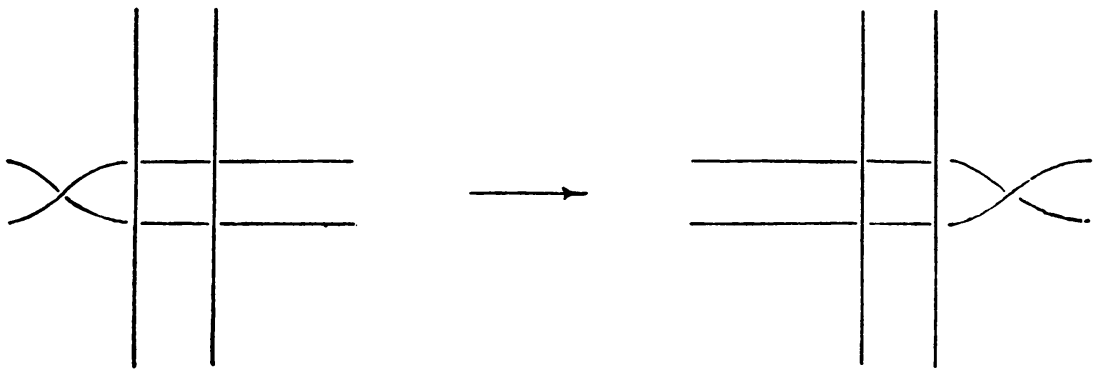

Case I. A twisted portion of the ribbon passes under (over) another portion of the ribbon.
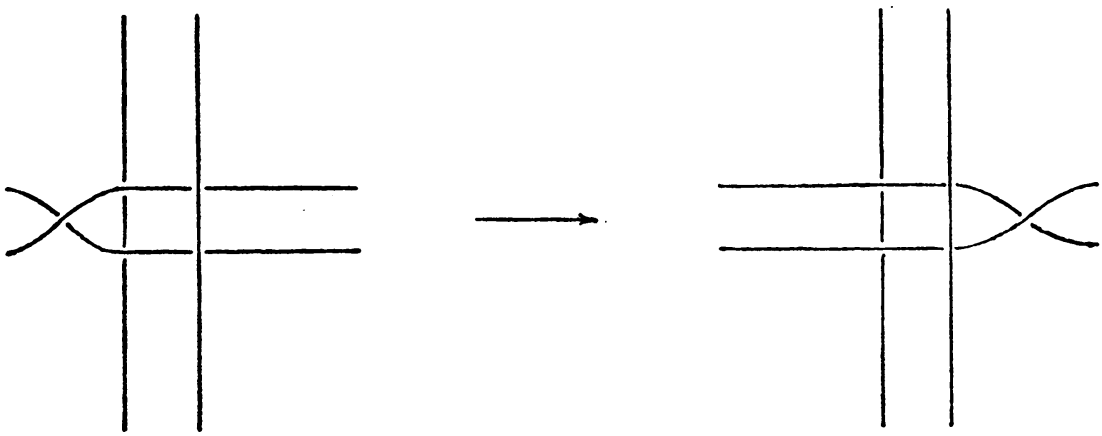

Case II. A twisted portion of the ribbon passes through another portion of the ribbon.
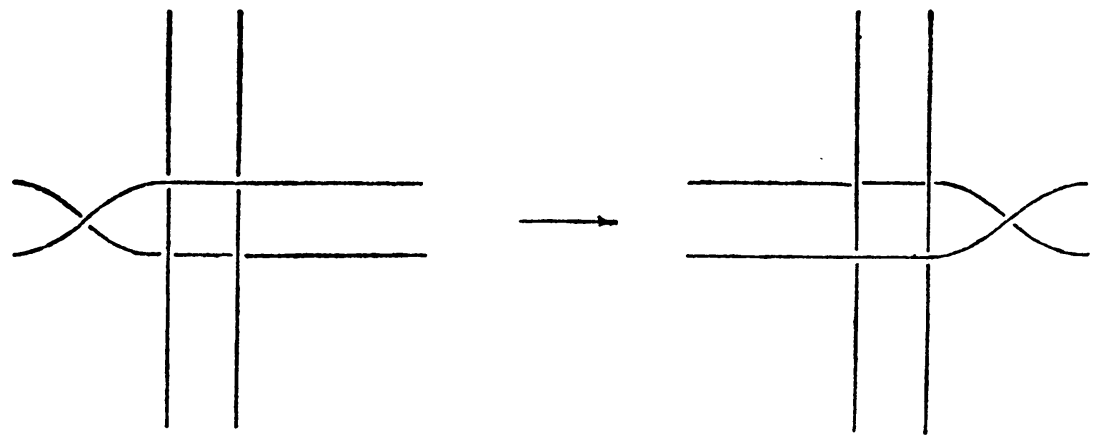

Case III. A portion of the ribbon passes through a twisted portion of the ribbon. 

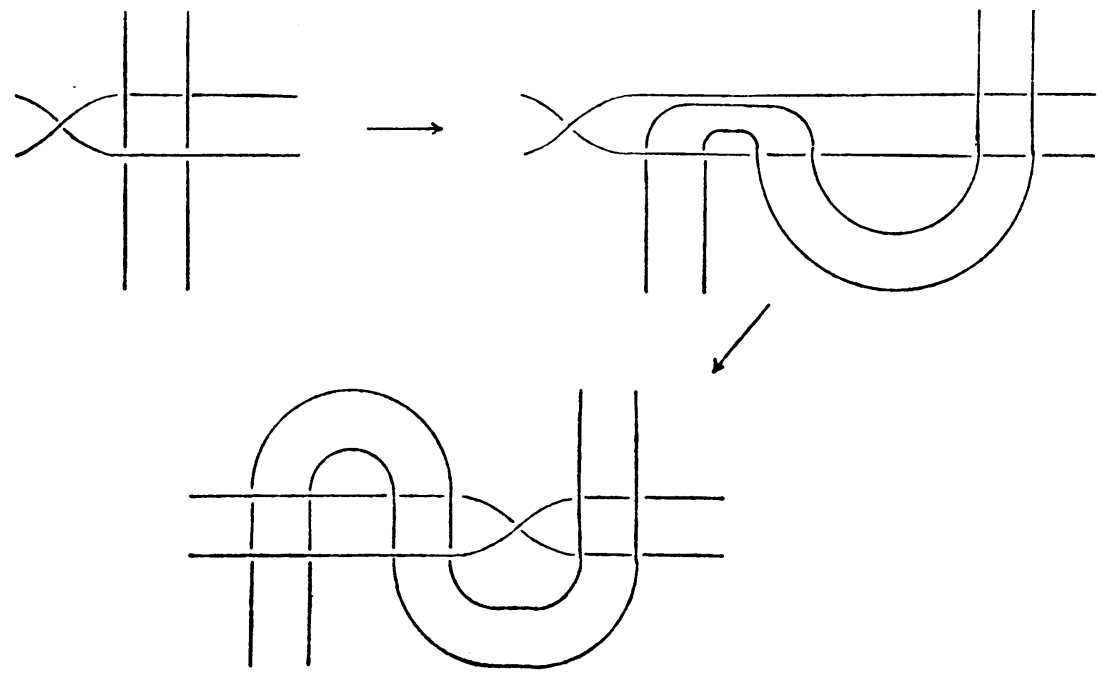

Case IV. A portion of the ribbon passes through a twisted portion of the ribbon in a manner different from Case III. The diagram shows how this case can be reduced to Case I.

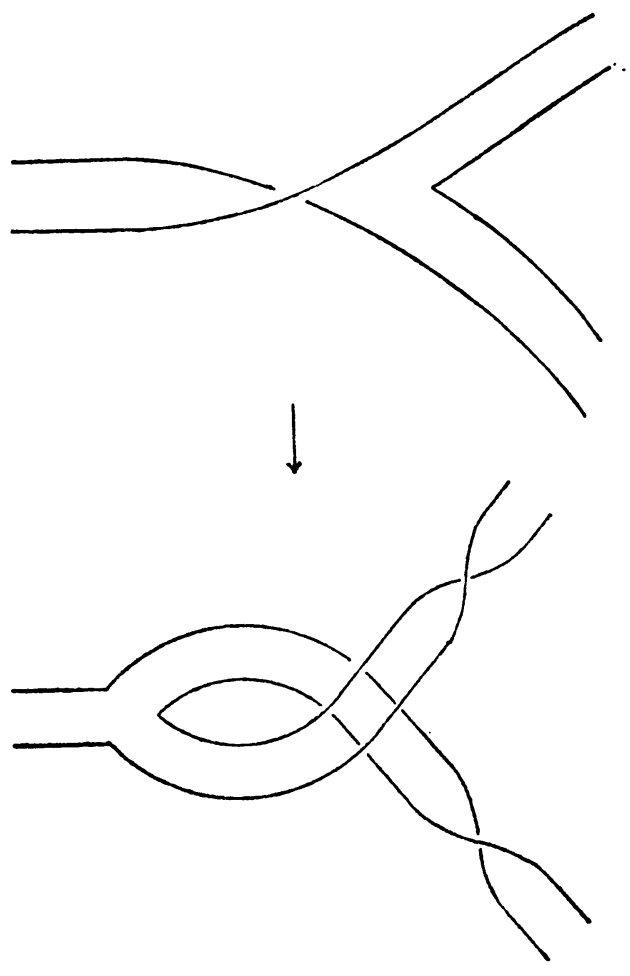


Case V. A twist is moved past a "fork" in the ribbon. In all of these cases a twist of the form

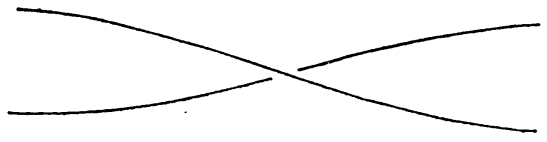

can be handled similarly.

3. Minkowski units. Let $k$ be a ribbon knot and let $P$ be a twistfree ribbon projection of $k$. In order to compute the Minkowski units [2, p. 28] for $k$, we first construct the quadratic matrix [2, p. 20] for $P$. We take a Reidemeister shading of the projection plane in which the unbounded region is white. Then the portion of the plane which is the projection image of the ribbon will be black, except for those places at which the ribbon crosses itself. See Figure 4.

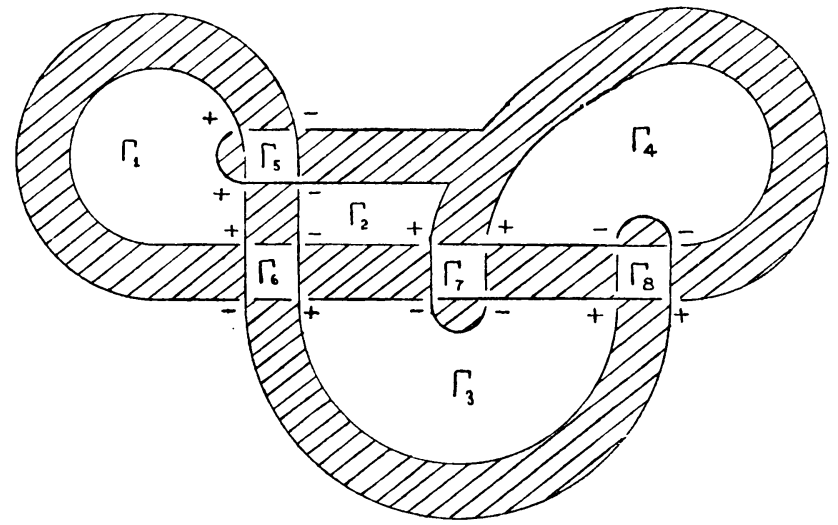

Figure 4

Take counterclockwise to be the direction of positive orientation of the plane, and assign to each doublepoint the index +1 or -1 according as the arc must be rotated in the positive direction over a white region or a black region to coincide with the lower arc. The white regions formed by the ribbon crossing itself will be called square regions, and the other bounded white regions will be called irregular regions.

REMARK A. The number of square regions is equal to the number of irregular regions.

Proof. If $g: I^{2} \rightarrow R^{3}$ is a ribbon, and if $P: R^{3} \rightarrow R^{2}$ gives a twist-free ribbon projection of $g\left(\dot{I}^{2}\right)$, then $P\left(g\left(I^{2}\right)\right)$ can be shrunk to the singular tree $P(g(T))$, where $T$ is the tree in $I^{2}$ described in the proof of Lemma 1 . In this shrinking, the number of irregular regions remains 
the same and the square regions become singular points (doublepoints) in the mapping $P \circ g: T \rightarrow R^{2}$. Thus to prove the remark we need only show that the number $\alpha$ of doublepoints in $P(g(T))$ is equal to the number $\beta$ of bounded regions in $R^{2}-P(g(T))$. Let $a$ be the number of vertices in $T$ and let $b$ be the number of edges in $T$. By the Euler formula we have

$$
a-b+1=2 .
$$

The number of vertices in $P(g(T))$ is $\alpha+a$, and it is fairly easy to see that the number of edges in $P(g(T))$ is $b+2 \alpha$. Hence applying the Euler formula to $P(g(T))$ gives

$$
(\alpha+a)-(b+2 \alpha)+(\beta+1)=2 .
$$

By use of (1), equation (2) reduces to

$$
\alpha=\beta
$$

and this proves the remark.

REMARK B. The sum of the indices of the doublepoints around a square region is zero.

Proof. There are only two kinds of square regions possible, one kind obtained by the ribbon passing over itself and the other kind obtained by the ribbon passing through itself. The remark holds in both cases as can be verified by referring to Figure 4 .

REMARK C. No two irregular regions have a doublepoint in common (because the irregular regions are separated from each other by the image of the ribbon).

REMARK D. No two square regions have a doublepoint in common (because the square regions are separated from each other by images of sections of the ribbon).

By Remark A we can let $\Gamma_{1}, \cdots, \Gamma_{n}$ be the irregular regions and $\Gamma_{n+1}, \cdots, \Gamma_{2 n}$ be the square regions. The quadratic matrix $M=\left(a_{i j}\right)$ has a row corresponding to each bounded white region. In the $k$ th row $(k=1, \cdots, 2 n), a_{k k}$ is the sum of the indices of the doublepoints around $\Gamma_{k}$ and $a_{k j}, j \neq k$, is the negative of the sum of the indices of the doublepoints shared by $\Gamma_{k}$ and $\Gamma_{j}$. Using Remarks B, C, and D, it is easy to see that the matrix $M$ obtained from $P$ has the form

$$
M=\left[\begin{array}{ll}
D & B \\
B^{t} & 0
\end{array}\right]
$$

where $D$ is an $n \times n$ diagonal matrix, 0 is the $n \times n$ zero-matrix, $B$ is an $n \times n$ matrix, and $B^{t}$ is the transpose of $B$. 
It is known that the quadratic matrix is always nonsingular. From (1) it is evident that

$$
\operatorname{det} M=(-1)^{n} \operatorname{det} B \cdot \operatorname{det} B^{t},
$$

and from this it follows that $\operatorname{det} B \neq 0$, so $B$ is also nonsingular.

Letting $R$ be the matrix

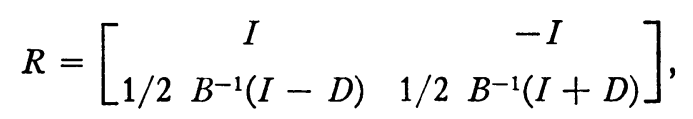

we see that ${ }^{2}$

$$
R^{t} M R=\left[\begin{array}{rr}
I & 0 \\
0 & -I
\end{array}\right]
$$

Since $R$ is a rational matrix, and since the Minkowski units of a matrix are preserved under rational transformations, it follows that $M$ has the same Minkowski units as $R^{t} M R$. But

$$
\operatorname{det} R^{t} M R= \pm 1 \text {, }
$$

so no odd prime divides det $R^{t} M R$. Hence for each odd prime $p$, the Minkowski unit $C_{p}$ is +1 . We have thus proved

THEOREM 3. For a ribbon knot, the Minkowski units $C_{p}$ are +1 for all odd primes $p$.

\section{RETERENCES}

1. R. H. Fox, Some problems in knot theory, Topology of 3-manifolds symposium, Prentice-Hall, Englewood Cliffs, N. J., 1962.

2. K. Reidemeister, Knotentheorie, Vol. 1, Ergebnisse der Mathematik und ihrer Grenzgebiete, Springer, Berlin, 1932.

3. J. H. C. Whitehead, Simplicial spaces, nuclei and m-groups, Proc. London Math. Soc. 45 (1939), 243-327.

Florida State University and

Florida Presbyterian College

2 The authors are indebted to Marion Tinsley and the referee for valuable assistance in obtaining this transformation. 